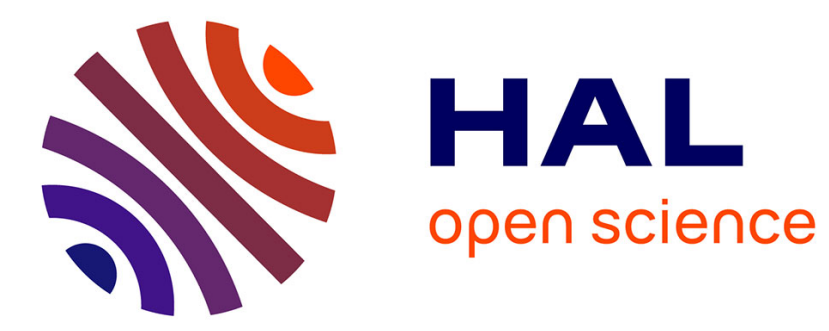

\title{
Relationship between the morphology and the light scattering cross section of optically soft aggregates Frédéric Gruy
}

\section{To cite this version:}

Frédéric Gruy. Relationship between the morphology and the light scattering cross section of optically soft aggregates. Journal of Quantitative Spectroscopy and Radiative Transfer, 2011, 112 (16), pp.26092618. 10.1016/j.jqsrt.2011.07.001 . hal-00619052

\section{HAL Id: hal-00619052 https://hal.science/hal-00619052}

Submitted on 5 Sep 2011

HAL is a multi-disciplinary open access archive for the deposit and dissemination of scientific research documents, whether they are published or not. The documents may come from teaching and research institutions in France or abroad, or from public or private research centers.
L'archive ouverte pluridisciplinaire HAL, est destinée au dépôt et à la diffusion de documents scientifiques de niveau recherche, publiés ou non, émanant des établissements d'enseignement et de recherche français ou étrangers, des laboratoires publics ou privés. 


\title{
RELATIONSHIP BETWEEN THE MORPHOLOGY AND THE LIGHT SCATTERING CROSS SECTION OF OPTICALLY SOFT AGGREGATES
}

\author{
GRUY Frédéric \\ Ecole des Mines, 158 Cours Fau riel, 42023 Saint-Etienne, Cedex 2 \\ gruy@emse.fr
}

\begin{abstract}
The purpose of this paper is to establish the suitable analytical expressions for the light scattering cross section of aggregates constituted by optically soft primary particles. The used optical approximation is the well-known Rayleigh-Debye-Gans approximation. The geometrical description of the aggregate is based on the pair, or distance, distribution density function describing at once the two spatial scales (primary particle and aggregate). Firstly, a study of the two-particle set is presented. An exact and an approximated formulations are proposed for the distance distribution density and the scattering cross section. Then the corresponding results are extended to the aggregates.
\end{abstract}

Keywords: aggregate, light scattering, pair distribution density, Rayleigh-Debye-Gans approximation

\section{Introduction}

Micro-particles mostly appear as aggregates constituted by nanometric or micrometric primary particles. Their geometrical and physical properties are the subject of numerous studies. An extensive literature exists for a particular class of aggregates known as the fractal-like aggregates. Filippov et al. studied the relationship between the morphology and the thermal behaviour of fractal aggregates [1]. The determination of their optical properties is particularly important also. The optical properties are needed not only for the characterization of micro-particles in a large number of industrial processes, but also for the study of environmental problems. Sorensen [2] has deeply studied the scattering properties of fractal aggregates.

During the monitoring of particle aggregation in an industrial process, the optical properties of the suspension are measured by means of an experimental set-up consisting of a mono- or polychromatic light source, a sampling cell and a photodetector. The incident light is scattered by the sample, i.e. the suspension. Then, one can record either the light intensity $I(\vartheta)$ at various scattering angles $\vartheta$ or the transmitted intensity, i.e. the non-scattered and non-absorbed light. The transmitted light is easier to work up. However the transmitted light intensity only leads to the light extinction cross section, that is $\int I(\vartheta) \sin \vartheta d \vartheta$. So, the light extinction cross section is obtained through an attenuation measurement by means of the Beer-Lambert law for 
diluted suspension. The measurement is performed at various wavelengths. This method has been used for the study of the aggregation of silica micro-particles [3] and polystyrene beads [4].

Simulations show that the aggregation of mono-sized primary particles results in a fractal-like cluster characterized by its fractal dimension. This morphological property is clearly proved for aggregates with a large number of primary particles $\left(\mathrm{N}>10^{3}\right)$. All geometrical properties of a fractal cluster can be deduced from its radius, its number of primary particles and its fractal dimension. Nevertheless, in real case the aggregates contain a small number of primary particles $\left(\mathrm{N}<<10^{3}\right)$ due to hydrodynamic and physicochemical conditions occurring in chemical reactors, e.g. high shear flow. Such aggregates are not as fractal-like ones.

Kahnert [5] reviewed the exact theories and numerical methods about the calculation of the optical properties of non spherical particles and aggregates. One can classify them into three main categories:

- Methods based on the partial differential equations that calculate the scattering field by solving the Maxwell equations or the Helmholtz equation. They are subjected to the boundary conditions suitable in the time or the frequency domain.

- Methods based on integration over volume or surface of equations derived from the Maxwell equations. Thus the boundary conditions are automatically included in the solution.

- The other methods are known as hybrids since they derive from the various approaches.

Among the first category, T-matrix method is widely used. In the T-matrix method, the incident and scattering fields are expressed in the form of a series of spherical vector wave functions. The expansion coefficients of the incident wave and the scattering wave are connected by a linear transformation. The corresponding matrix $\mathrm{T}$ contains all the information on the particle's optical properties for a given wavelength. It is a function of the size parameter, the shape, the optical refractive index of the considered particles, but it does not depend on the incident field. Thus this matrix does not need to be computed at each particle orientation change or change of the incident wave direction.

To classify the publications relying on this method, a database was carried out by Mishchenko et al.[6-9].

The solving by separation of variables for only one sphere can be enlarged to an aggregate of spheres by using the translation theorem for the spherical wave vector functions which expresses them in various bases of coordinates, and by applying a superposition principle. The total scattering field for an aggregate is then represented by the superposition of all the individual scattering fields resulting from each particle knowing that these fields are interdependent. Moreover, the problem can be formulated in the way of a T-matrix. A particular case of the T-matrix method [6] bearing the name of GMM (Generalized Multi-particle Mie-solution) is particularly suitable for sphere aggregates. The details of GMM are given by $\mathrm{Xu}[10,11]$. This method is very precise but its computation time depends on the number and the size parameter of primary particles.

However the study of the aggregation process, and the prediction of the time course of the turbidity spectrum require a too large computational time. Thus simple and accurate expressions for the optical properties of aggregates are needed. Simplified methods were developed for predicting the scattering and absorption cross sections of aggregates for several practical situations [12-15].

As above-mentioned, fractal-like aggregates are not so frequent during the aggregation process. As a consequence other geometrical parameters have to be chosen for 
quantifying the aggregate morphology. Among the geometrical parameters describing any body, the distribution density functions relative to some quantities are involved in the calculation of scattering parameters. Among these quantities, one can find:

- The chord length, e.g. the distance between two points located on the surface of a convex body [16]

- The distance between two points located inside the body [17]

- The pair (distance), i.e. the distance between the centres of any two primary particles in a cluster [2]

- The projected area [18].

These functions can often be analytically calculated for convex bodies. However, the calculation is less easy for non convex bodies [19, 20], as clusters of primary particles.

The choice of the relevant geometrical parameter depends on the size and the relative refractive index $m ; m$ is defined by the ratio between the refractive indices of the particle and the surrounding medium. Some scatterers dispersed in a liquid are characterized by a low optical contrast, i.e. $|m-1|<<1$. For instance, minerals like amorphous silica, silicates, carbonates, carboxylates, ice and gas hydrate crystals in water are optically soft. Likewise, the biological cells or microorganisms in water behave so. A number of important analytical approximations have been obtained for such particles. Rayleigh-Debye-Gans (RDG) approximation considers small soft particles with small phase shift, namely $[21,22]$ :

$2 \pi d / \lambda|m-1|<<1$

where $d$ and $\lambda$ are respectively the particle size and the light wavelength. The use of RDG approximation for fractal clusters led to numerous works, because it is suitable for explaining small angle scattering experiments with $\mathrm{X}$ ray or light. So the fractal dimension of aggregates can be deduced from the scattering data [23], because there exists a mathematically tractable relation between the scattered light intensity, the scattering wave vector and the fractal dimension. The ground is a particularly simple expression for the pair distribution density function for the fractal clusters. This analysis has been criticized by some authors, because it was used with non optically soft materials [24]. Furthermore a more realistic pair distribution density function of fractal clusters needs the insertion of a cut-off function [2].

The aim of this paper is to calculate the light scattering cross section of an optically soft aggregate from a distance distribution density. This will take into account at once the aggregate structure and the primary particle shape. Quasi-analytical expressions will be deduced and will be available for fast calculations.

The second section of this paper reminds the fundamentals about the RDG approximation and its relationship with the pair distribution density function. Section three presents some results concerning the distance distribution density function for a two-particle set with equal or different particle sizes. This section is completed by the study of the distance distribution density function for an aggregate. The section four is devoted to the exact calculation and an approximated one of the light scattering cross section for an aggregate with identical primary particles.

\section{Rayleigh-Debye-Gans approximation and aggregates}


Generally, the object (primary particle, aggregate ...) can be divided into smaller identical parts (elements). Each element is polarisable. In the presence of a variable electric field, the element becomes an oscillating dipole, which itself creates an electromagnetic field. When an object is illuminated by an electromagnetic wave, each element receives the incident electric field and the one coming from the other elements. As a result an oscillating dipole moment may be associated to each element. Thus the object emits an electromagnetic wave (scattered wave), which includes the contribution of each oscillating dipole.

In the Rayleigh-Debye-Gans domain, there is only interference of light waves that are independently scattered by all small volume elements. If the object is an aggregate, the element may be either the whole primary particle or a part of the primary particle.

Most often the incident wave is randomly polarised and the object (scatterer) can randomly orientate. The optical properties are thus obtained after calculating an average over all the wave polarisation states and object orientations.

The averaged light scattering cross section $C_{N}$ for aggregates consisting of $N$ primary particles with radius $a$ obeys the following relation:

$$
C_{N}=N^{2} \int_{(4 \pi)} F_{1}(\vartheta) S(Q) d \Omega \text { with } C_{1}=\int_{(4 \pi)} F_{1}(\vartheta) d \Omega
$$

where $\vartheta, \Omega, Q$ are respectively the scattering angle, the solid angle and the magnitude of the scattering wave vector $(Q=2 k \sin (\vartheta / 2) ; k=2 \pi / \lambda) . F_{1}(\vartheta)$ corresponds to the primary particle. The structure factor $S(Q)$ depends on the aggregate morphology [25]:

$$
S(Q)=\left[N+\sum_{i=j=1, i \neq j}^{N} \sin \left(Q R_{i j}\right) /\left(Q R_{i j}\right)\right] / N^{2}=\left[1+(N-1)\left\langle\sin \left(Q R_{i j}\right) /\left(Q R_{i j}\right)\right\rangle_{i j}\right] / N
$$

where $R_{i j}$ is the distance between the centres of the primary particles $i$ and $j .\langle\rangle_{i j}$ represents the averaged value over all $i, j$ pairs.

The scattering cross section of a doublet of primary particles separated by the centre to centre distance $2 a+h$ has been calculated for several materials and sizes of primary particle. The primary particles were considered as Rayleigh [26] or Mie [27-29] scatterers. For Rayleigh-Debye-Gans scatterers it is expressed as [21, 25]:

$$
C_{2} \simeq \frac{8}{9} \pi a^{2}(k a)^{4}(m-1)^{2} \int_{0}^{\pi} G^{2}(Q a)\left(1+\cos ^{2} \vartheta\right)\left(1+\frac{\sin (Q(2 a+h))}{Q(2 a+h)}\right) \sin \vartheta d \vartheta
$$

with

$$
G(u)=\frac{3}{u^{3}}(\sin u-u \cos u)
$$

In a continuous formulation, the structure factor can be written as:

$$
S(Q)=\int_{R_{\min }}^{R_{\max }} \sin (Q R) /(Q R) D_{P}(R) d R
$$

The distribution density function $D_{P}(R)$ for the distance $R$ between primary particle pair so appears. This function is chosen as a normalized one:

$\int_{R_{\min }}^{R_{\max }} D_{P}(R) d R=1$ 
where $R_{\min }$ and $R_{\max }$ are respectively the smallest and the largest distances between the primary particles.

Then the scattering cross section can be written as [30, 31]:

$$
C_{N}=N C_{1}\left[1+(N-1)\left\langle F^{R D G}\left(k R_{i j}\right)\right\rangle_{i j}\right] \underset{N \rightarrow \infty}{\simeq} N^{2} C_{1}\left\langle F^{R D G}(k R)\right\rangle
$$

The mean value in Eq.6 is expressed within a continuous formulation as:

$$
\langle X\rangle=\int_{R_{\min }}^{R_{\max }} X(R) D_{P}(R) d R
$$

with

$$
F^{R D G}(k a, R / a)=\int_{0}^{1} V(x, k a) \sin (2 k R x) /(2 k R x) d x / \int_{0}^{1} V(x, k a) d x
$$

and

$$
V(x, k a)=x\left(1+\left(1-2 x^{2}\right)^{2}\right)\left[\frac{\sin (2 k a x)}{(2 k a x)^{3}}-\frac{\cos (2 k a x)}{(2 k a x)^{2}}\right]^{2}
$$

When $k a \rightarrow 0$, Eq.8a becomes [31]:

$F_{0}^{R D G}(x)=3\left[\cos (2 x)\left(-1+5 x^{-2}-3 x^{-4}\right)+\sin (2 x)\left(2 x^{-1}-6 x^{-3}\right)+1+x^{-2}+3 x^{-4}\right] /\left(4 x^{2}\right)$

$F^{R D G}(k a, R / a)$ only depends on $k R$.

Eq. 6 can be rewritten by using only continuous variables:

$$
C_{N} \simeq \frac{2}{3 \pi} k^{4} V^{2}(m-1)^{2}\left\langle F_{0}^{R D G}(k R)\right\rangle
$$

$V$ is the matter volume of the scattering body. Thus the scattering cross section is expressed as a function of the distribution density of the distance $R$ between any two points belonging to the scattering body. The latter will be merely called pair distribution density. Eq.10 is easily verified for a sphere when compared to the expression given by Van de Hulst [21]. It is valid for any scattering body.

\section{Pair or distance distribution density function}

\subsection{Doublet of identical primary particles}

Let us examine the case of a doublet of two identical spheres with radius $b$. Figure 1 represents the normalized pair or distance distribution density obtained by simulation. The distance $H$ is the distance between the centres of the two primary particles. $R$ is the distance between two points inside the doublet. All distances are made dimensionless with regard to the radius of the primary particle.

The pair distribution density function for a doublet contains two contributions:

$D p(R, H)=\left(D p_{1 s}(R)+D p_{2 s}(R, H)\right) / 2$

$D p_{1 s}(R)$ and $D p_{2 s}(R, H)$ are respectively the normalized pair distribution density for a single sphere (the two points of the segment are inside the same sphere) and for a doublet (each end of the segment belongs to different spheres). $D p_{2 s}(R, H)$ will be called the partial pair distribution density. $D p(R, H)$ is also normalized.

The pair distribution density for a sphere [17] obeys:

$D p_{1 s}(R)=\frac{3}{16} R^{2}\left(R^{3}-12 R+16\right)$ 
for $0<R<2$.

The distribution $j$ th-order moments are:

$M_{1 s, j}=12 \times 2^{j}\left[\frac{1}{6+j}-\frac{3}{4+j}+\frac{2}{3+j}\right]$

The partial pair distribution density is such as:

$D p_{2 s}(R, H)=A \int_{T_{1}}^{T_{2}} S_{1} S_{2} d R^{\prime}$

, where $A$ is the normalization constant.

The notations are illustrated on Figure 2. A simple calculation leads to:

if $R<H$

$$
\begin{aligned}
& D p_{2 s}^{-}(R, H)=A \pi^{2} \frac{R}{H} \int_{H-1}^{R+1}\left(1-R+R^{\prime}\right)\left(1+R-R^{\prime}\right)\left(1-H+R^{\prime}\right)\left(1+H-R^{\prime}\right) d R^{\prime} \\
& D p_{2 s}^{-}(R, H)=\frac{3}{160} \frac{R}{H}(R-H+2)^{3}\left((R-H)^{2}-6(R-H)+4\right)
\end{aligned}
$$

if $R>H$

$$
\begin{aligned}
& D p_{2 s}^{+}(R, H)=A \pi^{2} \frac{R}{H} \int_{R-1}^{H+1}\left(1-R+R^{\prime}\right)\left(1+R-R^{\prime}\right)\left(1-H+R^{\prime}\right)\left(1+H-R^{\prime}\right) d R^{\prime} \\
& D p_{2 s}^{+}(R, H)=\frac{3}{160} \frac{R}{H}(H-R+2)^{3}\left((R-H)^{2}+6(R-H)+4\right)
\end{aligned}
$$

The $j$-moments of the partial pair distribution (doublet) $D p_{2 s}(R, H)$ are:

$$
M_{2 s, j}=F_{2 s, j}^{-}(H, H)-F_{2 s, j}^{-}(H-2, H)+F_{2 s, j}^{+}(H+2, H)-F_{2 s, j}^{+}(H, H)
$$

with

$$
F_{2 s, j}^{ \pm}(R, H)=\frac{3}{160 H} \sum_{q=0}^{j+1} C_{j+1}^{q}(H \pm 2)^{j+1-q}\left[\mp \frac{(R-H \mp 2)^{6+q}}{6+q}-10 \frac{(R-H \mp 2)^{5+q}}{5+q} \mp 20 \frac{(R-H \mp 2)^{4+q}}{4+q}\right]
$$

It can be deduced:

$$
M_{2 s, j}=\sum_{m=0}^{j+1} B_{m, j} H^{m-1}
$$

with

$$
\begin{aligned}
& B_{m, j}=0 \quad \text { if } \quad j+1-m \quad \text { is odd } \\
& B_{m, j}=\frac{3}{160} \sum_{q=0}^{j+1-m}(-1)^{q} C_{j+1}^{q} C_{j+1-q}^{m} 2^{j+8-m}\left((6+q)^{-1}-5(5+q)^{-1}+5(4+q)^{-1}\right) \quad \text { if } \quad j+1-m \text { is even }
\end{aligned}
$$

As an extension,

$$
B_{m, j}=0 \quad \text { if } \quad m>j+1
$$

If the set of the two spheres separated by the $H$ distance is converted to a set of two points separated by the same distance, then $D p_{2 s}(R, H)=\delta(R-H)$ and $M_{2 s, j}=H^{j}$, where $\delta$ is the Dirac function. Thus the $B_{m, j}$ coefficients represent the contribution of the primary particle shape (here a sphere) to the moments of the partial pair 
distribution density. The table 1 contains the $B_{m, j}$ coefficient values for the four first moments of the $D p_{2 s}$ density.

\subsection{Doublet of different primary particles}

Let us consider an asymmetric doublet consisting of spheres with radii $b_{1}$ and $b_{2}$ (with $b_{1}>b_{2}$ ). The distances are not made dimensionless because the choice of a characteristic length is not imperative.

The pair distribution density for an asymmetric doublet separated by the centre to centre distance $\mathrm{H}$ obeys the relation:

$D p(R, H)=P_{11} D p_{11}(R)+P_{22} D p_{22}(R)+2 P_{12} D p_{12}(R, H)$

The weighted coefficients $P_{i j}$ are simply:

$P_{11}=P_{1}^{2} \quad P_{22}=P_{2}^{2} \quad P_{12}=P_{1} P_{2} \quad$ with $\quad P_{j}=b_{j}^{3} /\left(b_{1}^{3}+b_{2}^{3}\right)$

$D p_{j j}(R)(j=1,2)$ and $D p_{12}(R, H)$ are respectively the normalized pair distribution densities for a single sphere (the two ends of the segment are inside the $j$ sphere) and for a doublet (each end of the segment belongs to different spheres). $D p_{12}(R, H)$ will be called the partial pair distribution density too.

The pair distribution density function for a single sphere is:

$$
D p_{j j}(R)=\frac{3}{16 b_{j}}\left(R / b_{j}\right)^{2}\left(\left(R / b_{j}\right)^{3}-12\left(R / b_{j}\right)+16\right)
$$

with $0<R<2 b_{j}$.

The partial distribution density for the doublet is also obtained from Eq.13a:

$$
D p_{12}(R, H)=A \pi^{2} \frac{R}{H} \int_{T_{1}}^{T_{2}}\left(b_{2}-R+R^{\prime}\right)\left(b_{2}+R-R^{\prime}\right)\left(b_{1}-H+R^{\prime}\right)\left(b_{1}+H-R^{\prime}\right) d R^{\prime}
$$

The normalization factor $A$ can be given by:

$$
A=\left(\frac{3}{4 \pi}\right)^{2}\left(b_{1} b_{2}\right)^{-3}
$$

The lower and upper limits $\mathrm{T}_{1}$ and $\mathrm{T}_{2}$ of the integral are:

$$
\begin{array}{lll}
\text { if } R<H+b_{2}-b_{1} & , T_{1}=H-b_{1} & T_{2}=R+b_{2} \\
\text { if } H+b_{2}-b_{1}<R<H+b_{1}-b_{2} & , T_{1}=R-b_{2} & T_{2}=R+b_{2} \\
\text { if } H+b_{1}-b_{2}<R<H+b_{1}+b_{2} & , T_{1}=R-b_{2} & T_{2}=H+b_{1}
\end{array}
$$

An analytical solution of this integral (Eq.19) exists as:

$$
D p_{12}=f\left(T_{2}-\beta\right)-f\left(T_{1}-\beta\right)
$$

where

$$
f(y)=A \pi^{2} \frac{R}{H}\left[\begin{array}{l}
\left(b_{1}^{2} b_{2}^{2}-\left(b_{1}^{2}+b_{2}^{2}\right) \alpha^{2}+\alpha^{4}\right) y \\
+\left(b_{1}^{2}-b_{2}^{2}\right) \alpha y^{2}-\left(b_{1}^{2}+b_{2}^{2}+2 \alpha^{2}\right) y^{3} / 3+y^{5} / 5
\end{array}\right]
$$

and $\alpha=(R-H) / 2$ and $\beta=(R+H) / 2$ 


\subsection{Set of identical primary particles}

The generalization of the previous calculations to a chain of $N$ primary particles (Eq.22) and to an aggregate with $N$ primary particles (Eq.23) is straightforward:

$\begin{array}{ll}D p(R)=\left(N D p_{1 s}(R)+\sum_{j=2}^{N} 2(N+1-j) D p_{2 s}(R, 2(j-1))\right) / N^{2} & \\ D p(R)=\frac{1}{N} D p_{1 s}(R)+\frac{N-1}{N} \int_{H_{\min }}^{H_{\max }} D p_{N e}(H) D p_{2 s}(R, H) d H & N \geq 1\end{array}$

$D p_{N e}(H)$ is the pair distribution density function for the set of the $N$ sphere centres. This set represents the superstructure of the body whereas the spherical primary particle represents its substructure. $D p_{N e}(H)$ is normalized. For a chain of $N$ primary particles, $D p_{N e}(H)$ obeys the expression:

$$
D p_{N e}(H)=\sum_{j=2}^{N} \frac{2 N-H}{N(N-1)} \delta(H-2(j-1))
$$

Figure 3a represents the pair distribution density function for a chain of $N$ primary particles (Eqs.23, 24). The two size scales are clearly identified. Figure $3 b$ represents the pair distribution density function for a cubic packing of $N$ primary particles. The two size scales can be observed, but less distinctly.

Using the expressions for the distribution moments of a primary particle (Eq.12b) and for a doublet (Eq.16a), the $j^{\text {th }}$-moment of the aggregate distribution is written as:

$$
M_{j}=\frac{M_{1 s, j}}{N}+\frac{N-1}{N} \sum_{m=0}^{j+1} B_{m, j} Q_{m-1}
$$

where $Q_{i}$ is the $i^{\text {th }}$-moment of the superstructure distribution $D p_{N e}(H)$.

The aggregate of $N$ spheres can be compared to the cluster of $N$ points (centres of spheres) for which:

$$
D p(R)=D p_{N e}(R)=\sum_{i, k=1, i \neq k}^{N} \delta\left(R-H_{i k}\right) /\left(N^{2}-N\right) .
$$

Then

$$
M_{j}=Q_{j}=\sum_{i, k=1, i \neq k}^{N} H_{i k}^{j} /\left(N^{2}-N\right)
$$

where $M_{1 s, j}$ and $B_{m, j}$ characterize once more the primary particle shape, i.e. the substructure.

\subsection{Set of different primary particles}

The pair distribution density function for a set of $N$ primary particles with different radii $b_{j}$ can be written as:

$$
D p(R)=\sum_{j=1}^{N} P_{j}^{2} D p_{j j}(R)+\sum_{\substack{j, k=1 \\ j \neq k}}^{N} P_{j} P_{k} D p_{j k}\left(R, H_{j k}\right)
$$

with 
$P_{j}=b_{j}^{3} /\left(\sum_{i=1}^{N} b_{i}^{3}\right)$

$H_{j k}$ is the distance between the centres of the primary particles $j$ and $k . R$ and $H_{j k}$ are not made dimensionless. One can verify that $D p$ is normalized. $D p_{j k}\left(R, H_{j k}\right)$ is calculated from Eqs.20-21.

\section{Light scattering cross section}

\subsection{Doublet of identical primary particles}

\subsubsection{Exact calculation}

The scattering cross section of a doublet as a function of the primary particle size parameter $k b$ has been calculated by two ways:

- following Van de Hulst (Eq.3),

- following Eqs. 9-10 and the pair distribution density for a doublet (Eq.11)

The deviation between the two calculations is smaller than $10^{-3}$ within the whole range $[0,100]$ of the size parameter.

Figure 4 represents the ratio of the scattering cross sections of the doublet and the primary particle as a function of the primary particle size parameter $k b$ for various distances $H$. The ratio tends to 4 if $k b \rightarrow 0$ and to a value close to 2 if $k b \rightarrow \infty$. One can also observe the occurrence of two $k b$-ranges: $[0,3]$ and $[3,100]$ : as $k b>3$, the ratio has a constant value within $5 \%$. The latter one follows the approximate expression:

$C_{2} / C_{1}=2+0.25 e^{-0.85(H-2)^{0.7}}$

\subsubsection{Approximated calculation}

The function $F_{0}^{R D G}(x)$ can be approximated by a polynomial function allowing the introduction of the moments of the distribution. Figure 5 and Eq.28a show such an approximation.

$$
\begin{array}{ll}
F_{a p p}(x)=1-0.4 x^{2}+0.1 x^{3} & 0<x<\alpha \\
F_{a p p}(x)=0 & x>\alpha
\end{array}
$$

where $\alpha=3.0$

or

$$
\begin{array}{ll}
F_{a p p}(x)=1-0.4 x^{2}+0.1 x^{3} & 0<x<\alpha \\
F_{a p p}(x)=-0.0105+0.21 / x & x>\alpha
\end{array}
$$

where $\alpha=2.5$

If the approximation (Eq.28a) is applied, the distribution moments with orders 0,2 and 3 appear in the expression of the scattering cross section. In fact, only the partial or the truncated moments, called $\left(M_{j}^{T}, M_{1 s, j}^{T}, M_{2 s, j}^{T}\right)$, are involved due to the relation $F_{a p p}(x>\alpha)=0$. 
In the case of the partial moment, the integration interval $[A, B]$ in $M_{j}^{T}=\int_{A}^{B} R^{j} D_{p}(R) d R$ is included within $\left[\mathrm{R}_{\min }, \mathrm{R}_{\max }\right] . \mathrm{R}_{\min }$ and $\mathrm{R}_{\max }$ are respectively the smallest and the largest distances between two points belonging to the scattering body.

Then for a doublet and setting $u=\alpha(k b)^{-1}$ :

$M_{j}^{T}=\left(M_{1 s, j}^{T}+M_{2 s, j}^{T}\right) / 2$

with

$M_{2 s, j}^{T}=A^{-} F_{2 s, j}^{-}\left(Y^{-}, H\right)-B^{-} F_{2 s, j}^{-}(H-2, H)+A^{+} F_{2 s, j}^{+}\left(Y^{+}, H\right)-B^{+} F_{2 s, j}^{+}(H, H)$

where

$$
\begin{array}{ll}
\left(A^{-}, B^{-}, A^{+}, B^{+}\right)=(1,1,1,1) \text { and }\left(Y^{-}, Y^{+}\right)=(H, H+2) & \text { if } 2<H<u-2 \\
\left(A^{-}, B^{-}, A^{+}, B^{+}\right)=(1,1,1,1) \text { and }\left(Y^{-}, Y^{+}\right)=(H, u) & \text { if } u-2<H<u \\
\left(A^{-}, B^{-}, A^{+}, B^{+}\right)=(1,1,0,0) \text { and }\left(Y^{-}, Y^{+}\right)=(u, H) & \text { if } u<H<u+2 \\
\left(A^{-}, B^{-}, A^{+}, B^{+}\right)=(0,0,0,0) & \text { if } H>u+2
\end{array}
$$

and

$M_{1 s, j}^{T}=\int_{0}^{Y} R^{j} D p_{1 s} d R$

where

$Y=2$ if $u>2$

$Y=u$ if $u<2$.

However, Eq.31 (with $Y=u$ ) leads to an erroneous value for the moments of the distribution in the case of the large primary particles. As a consequence, one keeps out Eq.31 with $Y=2$, i.e. $M_{1 s, j}^{T}=M_{1 s, j}$.

Then, the scattering cross section of the doublet is written as:

$C_{2}(H)=\frac{2}{3 \pi} k^{4} V^{2}(m-1)^{2}\left(M_{0}^{T}-0.4 M_{2}^{T}+0.1 M_{3}^{T}\right)$

or

$$
C_{2}(H)=2 C_{1}+C_{2 p}(H)
$$

with

$$
C_{2 p}(H)=\frac{1}{3 \pi} k^{4} V^{2}(m-1)^{2}\left(M_{2 s, 0}^{T}-0.4 M_{2 s, 2}^{T}+0.1 M_{2 s, 3}^{T}\right)
$$

Figure 6 compares $C_{2}(H)$ calculated following Van de Hulst (Eq.3) and (Eqs.33ab). Only the doublet of touching primary particles $(H=2)$ leads to a non-negligible error for $k b>3$. The use of the second approximation (Eq.28b) leads to a negligible error for $k b<15$.

If the largest distance between two points of the doublet is such as $H+2<u$ (i.e., the largest distance is smaller than $\alpha / k \approx \lambda / 2)$, then Eq.14 and Eqs.16a-c can be applied for any $H$. Eq.33b becomes:

$$
C_{2 p}(H)=\frac{1}{3 \pi} k^{4} V^{2}(m-1)^{2}\left(1-\sum_{m=0}^{4}\left(0.4 B_{m, 2}-0.1 B_{m, 3}\right) H^{m-1}\right)
$$




\subsection{Set of identical primary particles}

The aggregate scattering cross section is calculated by using Eqs.10 and 23:

$$
C_{N}=N C_{1}+\frac{N(N-1)}{2} \int_{H_{\min }}^{H_{\max }} D p_{N e}(H) C_{2 p}(H) d H
$$

or

$$
C_{N}=N C_{1}+\sum_{\substack{j, k=1 \\ j \neq k}}^{N} C_{2 j k}\left(H_{j k}\right) / 2
$$

If the largest distance into the aggregate corresponds to the case where $\operatorname{Max}\left\{H_{j k}\right\}+2<u$ (i.e., the largest distance is smaller than $\alpha / k \approx \lambda / 2$ ), Eq.14 and 16a-c can be applied for all $H$. Eq.35b becomes:

$$
C_{N}=N C_{1}+\frac{N(N-1)}{2} \frac{1}{3 \pi} k^{4} V^{2}(m-1)^{2}\left(1-\sum_{m=0}^{4}\left(0.4 B_{m, 2}-0.1 B_{m, 3}\right) Q_{m-1}\right)
$$

$V$ is the matter volume of the doublet.

The percentage of doublets with primary particles touching each others $(H=2)$ in an aggregate is often small. The body having the largest ratio is a three-dimensional compact object. Although the deviation (Eq.3 vs Eqs.33a-b) previously observed for a doublet (figure 6) is small, the deviation for an aggregate is cumulative and increases with the number $N$ of primary particles.

The values of the ratio $C_{N} / N C_{1}$ have been calculated for various, $k b$ and $N$, values in the case of two aggregates: a simple cubic arrangement of primary particles and a chain of primary particles. The values of the ratio $C_{N} / N C_{1}$ were calculated by the following Eq.35a and by using either $F_{0}^{R D G}(x)$ or $F_{a p p}(x)$. After calculations the deviation between the two value sets is smaller than $5 \%$ if $k b N^{1 / d}<2.5$ for a $3 \mathrm{D}$ body $(d=3)$ and $k b N^{1 / d}<10$ for 1D-body $(d=1)$. In fact, $k b N^{1 / d}$ is the size parameter for the whole aggregate. Calculations were performed with $N<10^{4}$ for $d=3$ and $N<200$ for $d=1$. The condition $0<k b N^{1 / d}<2.5$ corresponds to a $\mathrm{x}$-range such as $F_{0}^{R D G}(x)=F_{\text {app }}(x)$ (see figure 5). This condition is strictly verified for a 3D-body whereas it is weakened for $1 \mathrm{D}$-body. In the latter case the ratio of primary particles separated by a large distance $(k R>2.5)$ is smaller than in a 3D-body and contribute less to the scattering cross section.

Figure 4 shows that for $k b>10$, the scattering cross section of the doublet does not depend on $k b$ and depends only on $H$. Eqs.27 and 35a lead to:

$$
C_{N}=N C_{1}\left[1+\frac{(N-1)}{2} \int_{H_{\min }}^{H_{\max }} D p_{N e}(H) 0.25 e^{-0.85(H-2)^{0.7}} d H\right]
$$

The deviation between the two value series (Eq.35a versus Eq.37) is also smaller than 5\%. The scattering cross section (Eq.37) depends on the whole morphology (superstructure) of the aggregate. The kernel of the integral (Eq.37) corresponds to the substructure, i.e. the primary particle.

Moreover, the scattering cross section for a set of different primary particles obeys: 


$$
C_{N}=\sum_{j=1}^{N} C_{1, j}+\sum_{\substack{j, k=1 \\ j \neq k}}^{N} C_{2 j k}\left(H_{j k}\right) 2 V_{j} V_{k} /\left(V_{j}+V_{k}\right)^{2}
$$

with

$$
\begin{aligned}
& C_{2 j k}=\frac{1}{3 \pi} k^{4}\left(V_{j}+V_{k}\right)^{2}(m-1)^{2} \int_{H_{j k}-b_{j}-b_{k}}^{H_{j k}+b_{j}+b_{k}} F_{0}^{R D G}(k R) D p_{j k}\left(R, H_{j k}\right) d R \\
& D p_{j k}\left(R, H_{j k}\right) \text { is calculated from Eqs.20-21. }
\end{aligned}
$$

\section{Conclusion}

An aggregate composed of spherical primary particles presents two size scales. In other words there exists a substructure (primary particle) and a superstructure (aggregate). The pair (distance) distribution density function shows the details of the two scales. These are more distinguishable from each other in the case of a body with a small spatial dimension like the chain of primary particles. The distribution $j^{\text {th }}$ order moment is a linear combination of the moments with order $\leq j$ of the superstructure distance distribution. The coefficients of the combination are relative to the sub-structure. In the framework of the RDG approximation, the scattering cross section of the aggregate is related to the distance distribution in the superstructure and the scattering cross section of a doublet. Equations are available as well as their approximations decreasing the computation time. However, there does not exist a relation between the scattering cross section of the aggregate and the distribution moments over the whole range of size and number of primary particles. This work will be enlarged to other primary particle shapes (e.g. polyhedral) in order to determine the corresponding combination coefficients in the $D p(R, H)$ moments and to calculate the doublet and aggregate scattering cross sections.

\section{References}

[1] Filippov A.V., Zurita M., Rosner D.E. Fractal-like aggregates: Relation between morphology and physical properties. Journal of Colloid and Interface Science 2000;229:261-273.

[2] Sorensen C.M. Light scattering by fractal aggregates: a Review. Aerosol Science and Technology 2001;35:648-687.

[3] Gruy F. Formation of small silica aggregates by turbulent aggregation. Journal of Colloid and Interface Science 2001;237:28-39.

[4] Xu S., Liu J., Sun Z. Optical factors determined by the T-matrix method in turbidity measurement of absolute coagulation rate constants. Journal of Colloid and Interface Science 2006;304:107-114.

[5] Kahnert F. M. Numerical methods in electromagnetic scattering theory. J. Quant. Spectr. Rad. Transfer 2003; 79(80): 775-824.

[6] Mishchenko M. I., Videen G., Babenko V.A., Khlebtsov N.G., Wriedt T. T-matrix theory of electromagnetic scattering by particles and its applications: a comprehensive reference database. J. Quant. Spectr. Rad. Transfer 2004; 88: 357-406.

[7] Mishchenko M. I., Videen G., Babenko V.A., Khlebtsov N.G., Wriedt T. Comprehensive T-matrix reference database: A 2004-06 update. J. Quant. Spectr. Rad. Transfer 2007; 106: 304-324. 
[8] Mishchenko, M. I., Videen G., Khlebtsov N.G., Wriedt T., Zakharova N.T. Comprehensive T-matrix reference database: A 2006-07 update. J. Quant. Spectr. Rad. Transfer 2008; 109: 1447-1460.

[9] Mishchenko, M. I., Zakharova N.T., Videen G., Khlebtsov N.G., Wriedt T. Comprehensive T-matrix reference database: A 2007-09 update. J. Quant. Spectr. Rad. Transfer 2010; 111: 650-658.

[10] Xu Y.-L. Electromagnetic scattering by an aggregate of spheres. Appl. Optics 1995; 34(21): 4573-4588.

[11] $\mathrm{Xu}$ Y.-L. Electromagnetic scattering by an aggregate of spheres: far field. Appl. Optics 1997; 36(36): 9496-9508.

[12] Mackowski D.W. A simplified model to predict the effects of aggregation on the absorption properties of soot particles. J. Quant. Spectr. Rad. Transfer 2006; 100: 237249.

[13] Jacquier, S., Gruy F. Approximation of the light scattering cross-section for aggregated spherical non-absorbent particles. J. Quant. Spectr. Rad. Transfer 2007;106: 133-144.

[14] Jacquier, S., Gruy F. Anomalous Diffraction Approximation for light scattering cross section: case of ordered clusters of non-absorbent spheres. J. Quant. Spectr. Rad. Transfer 2008; 109: 789-810.

[15] Jacquier, S., Gruy F. Anomalous Diffraction Approximation for light scattering cross section: case of random clusters of non-absorbent spheres. J. Quant. Spectr. Rad. Transfer 2008; 109: 2794-2803.

[16] Gille W. The small-angle scattering correlation function of the hemisphere. Computational Materials Science 1999;15:449-454.

[17] Gruy F. Light scattering cross section as a function of pair distribution density. J. Quant. Spectr. Rad. Transfer 2009;110:240-246.

[18] Jacquier S. Méthodes approchées pour les propriétés optiques d'agrégats de particules sphériques non absorbantes. PhD thesis;F-Saint-Etienne;2006

[19] Mazzolo A., Roesslinger B., Gille W. Properties of chord length distributions of nonconvex bodies. Journal of Mathematical Physics 2003;44:6195-6208.

[20] Gruy F., Jacquier S. The chord length distribution of a two-sphere aggregate. Computational Materials Science 2008;44:218-223.

[21] Van de Hulst H.C. Light scattering by small particles. NewYork: J. Wiley;1957.

[22] Kokhanovsky A.A. Optics of light scattering media: Problems and solutions. 2nd ed. Berlin: Springer-Praxis;2001.

[23] Hermawan M., Bushell G., Bickert G., Amal R. Characterization of short-range structure of silica aggregates-implication to sedimentation compaction. Int. J. Miner. Process 2004;73:65-81.

[24] Riefler N., di Stasio S., Wriedt T. Structural analysis of clusters using configurational and orientational averaging in light scattering analysis. J. Quant. Spectr. Rad. Transfer 2004;89:323-342.

[25] Khlebstov N.G. Spectroturbidimetry of fractal clusters: test of density correlation function cut-off. Applied Optics 1996;35:4261-4270.

[26] Lips A., Levine S. Light scattering by two spherical Rayleigh particles over all orientations. Journal of Colloid and Interface Science 1970; 33: 455-464

[27] Kattawar G.W., Dean C.E. Electromagnetic scattering for two dielectric spheres: comparison between theory and experiment. Optics Letters 1983; 8: 48-50

[28] Borghese F., Denti P., Saija R., Toscano G., Sindoni O.I. Macroscopic optical constants of a cloud of randomly oriented nonspherical scatterers. Il Nuovo Cimento 1984; 81B: 29-50

[29] Mishchenko M. I., Mackowski D. W., Travis L. D. Scattering of light by bispheres with touching and separated components, Appl. Optics 1995; 34: 4589-4599. 
[30] Berry M.V., Percival I.C. Optics of fractal clusters such as smoke. Optica Acta 1986;33:577-591.

[31] Gruy F. Relation entre morphologie et signature optique pour les particules nano et micrométriques. Récents Progrès en Génie des Procédés Paris:Ed. SFGPLavoisier;97;2008. 


\section{Captions}

Fig.1: Dp as a function of $R$ (normalized by the radius of the primary particle) for a doublet.

Fig. 2: drawing showing the notation and the procedure to calculate the pair distribution density; case of the doublet.

Fig.3a: $D p$ as a function of $R$ (made dimensionless by the radius of a primary particle) for a chain of $N$ primary particles.

Fig.3b: $D p$ as a function of $R$ (made dimensionless by the radius of a primary particle) for a cubic packing of $N$ primary particles.

Fig. 4: ratio between the scattering cross sections of the doublet and the primary particle for $H=2$ (---), $H=6$ ( _ $), H=12(+++)$ as a function of the primary particle size parameter.

Fig.5: $F_{0}^{R D G}(x)$ and $F_{a p p}(x)$ functions

Fig. 6: ratio between the scattering cross sections of the doublet and the primary particle as a function of the primary particle size parameter. Comparison between Van de Hulst (Eq.3) and this work (Eqs.33a-b). * $(H=1, E q .3), x(H=1$, Eqs.33a-b), o $(H=2, E q .3), .(H=2, E q s .33 a-b),<(H=5, E q .3),>(H=5, E q s .33 a-b)$ 


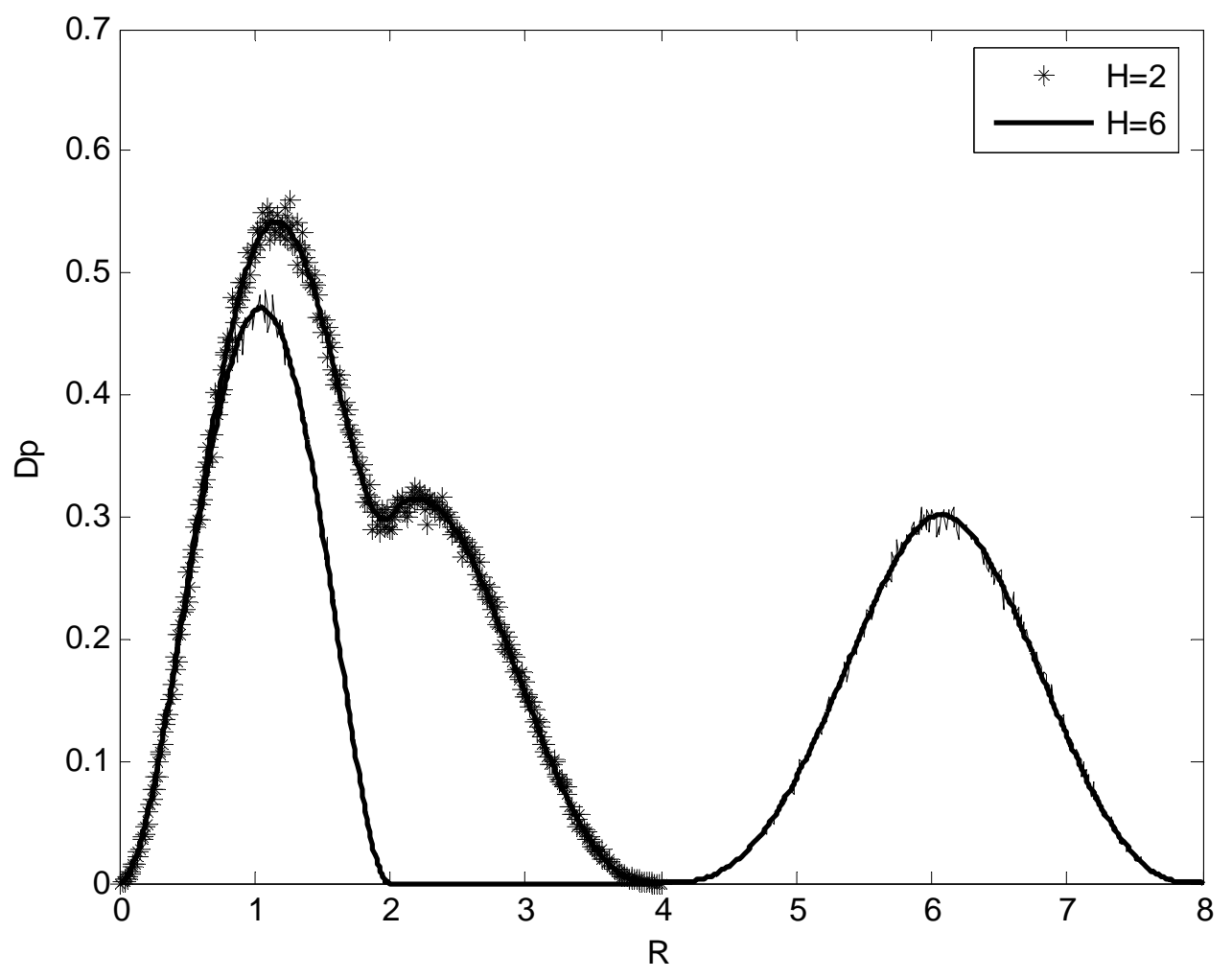

Fig.1 


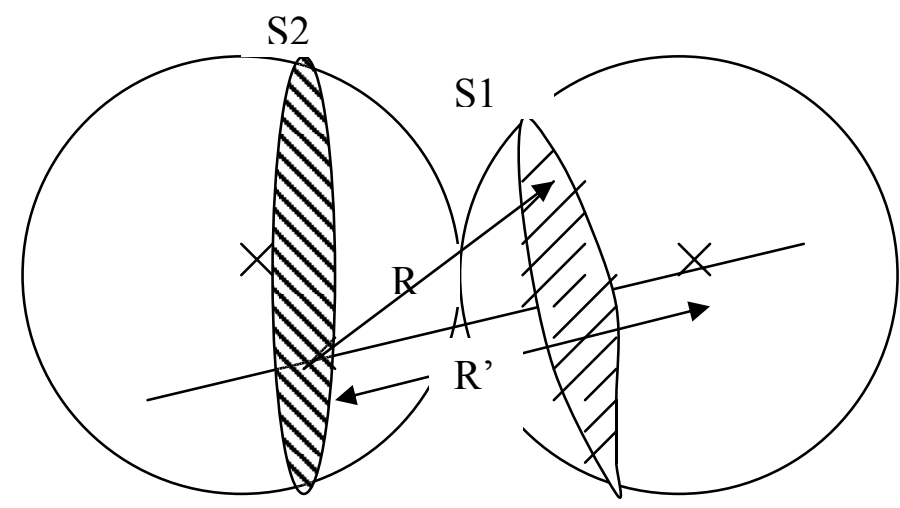

Fig. 2 


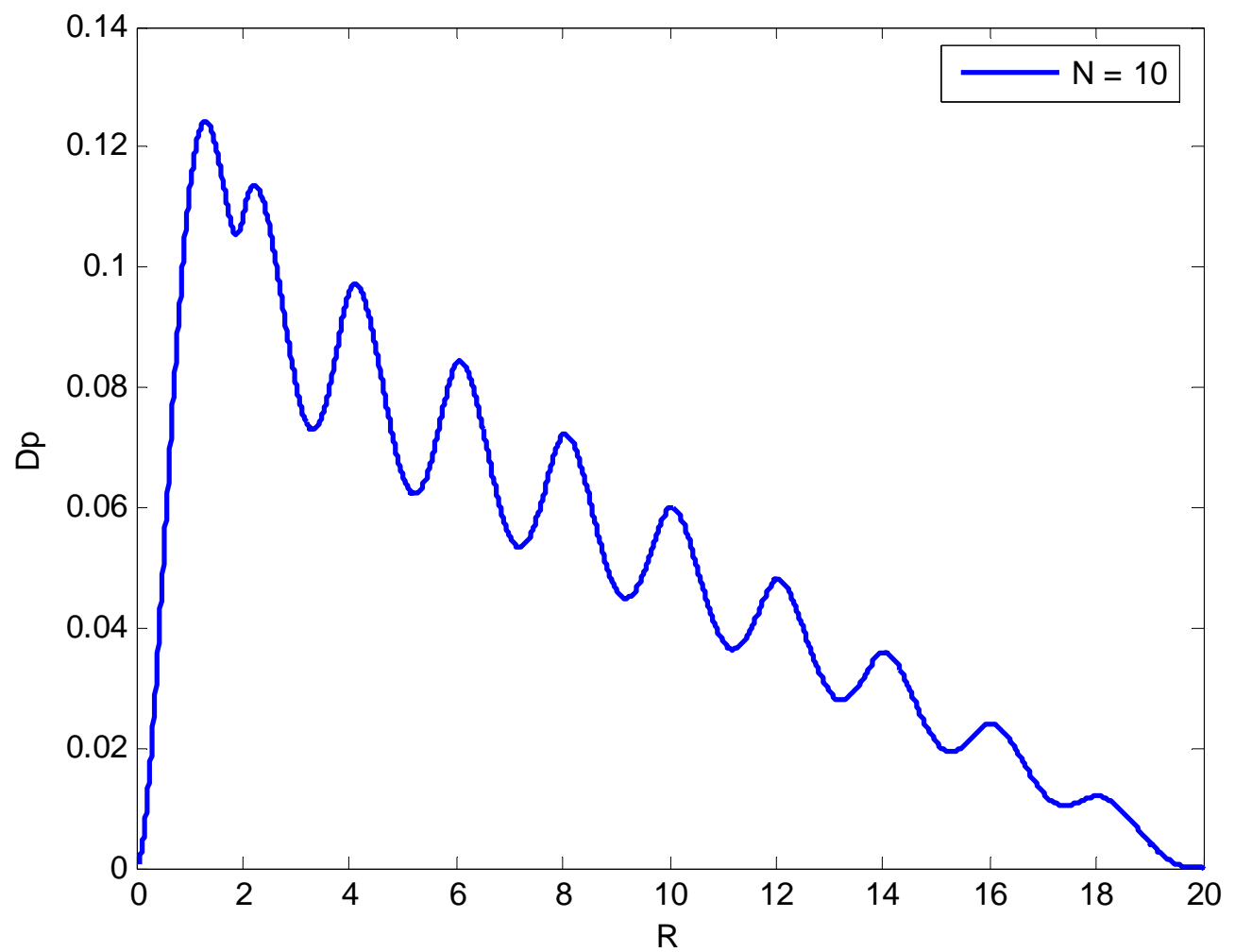

Fig.3a 


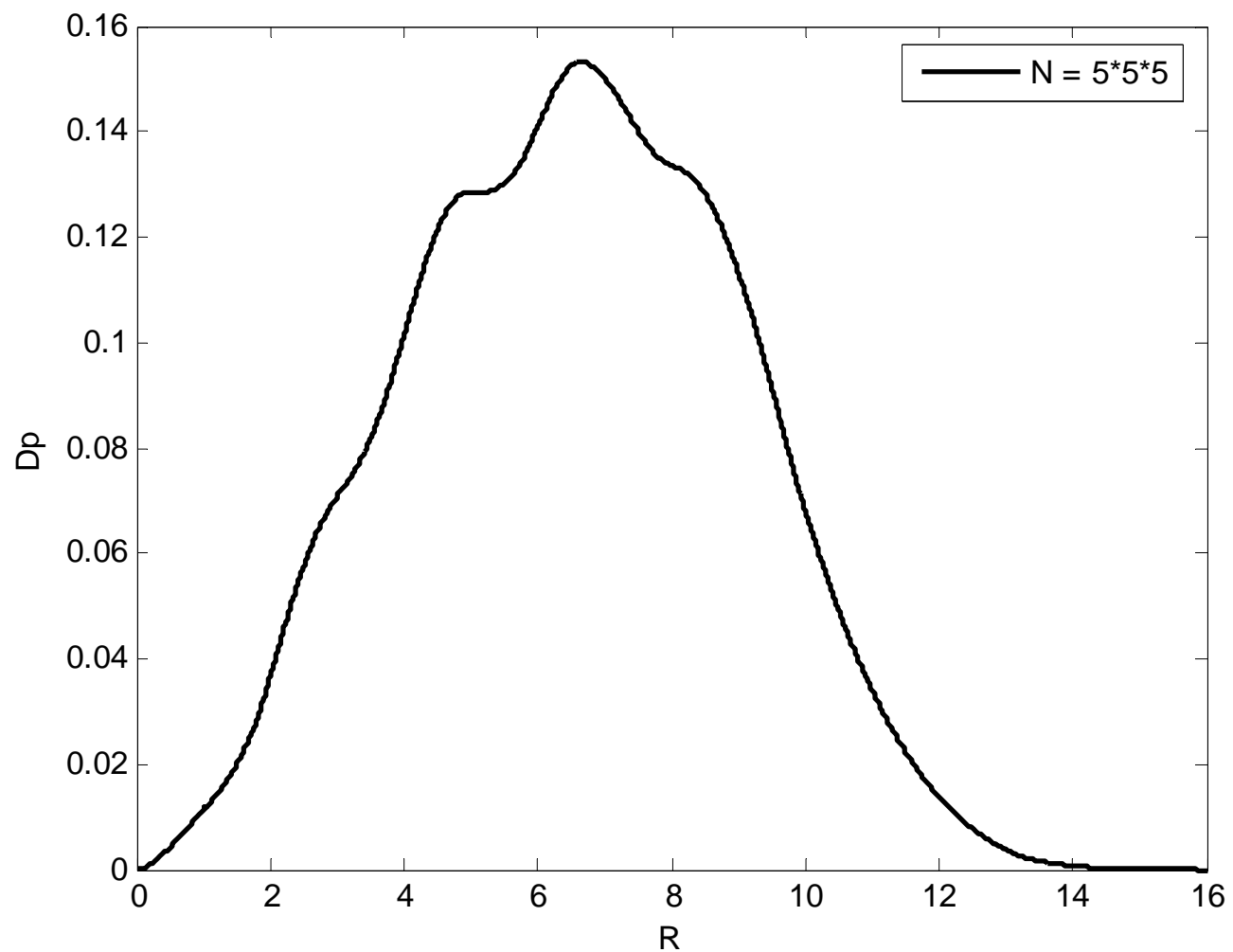

Fig.3b 


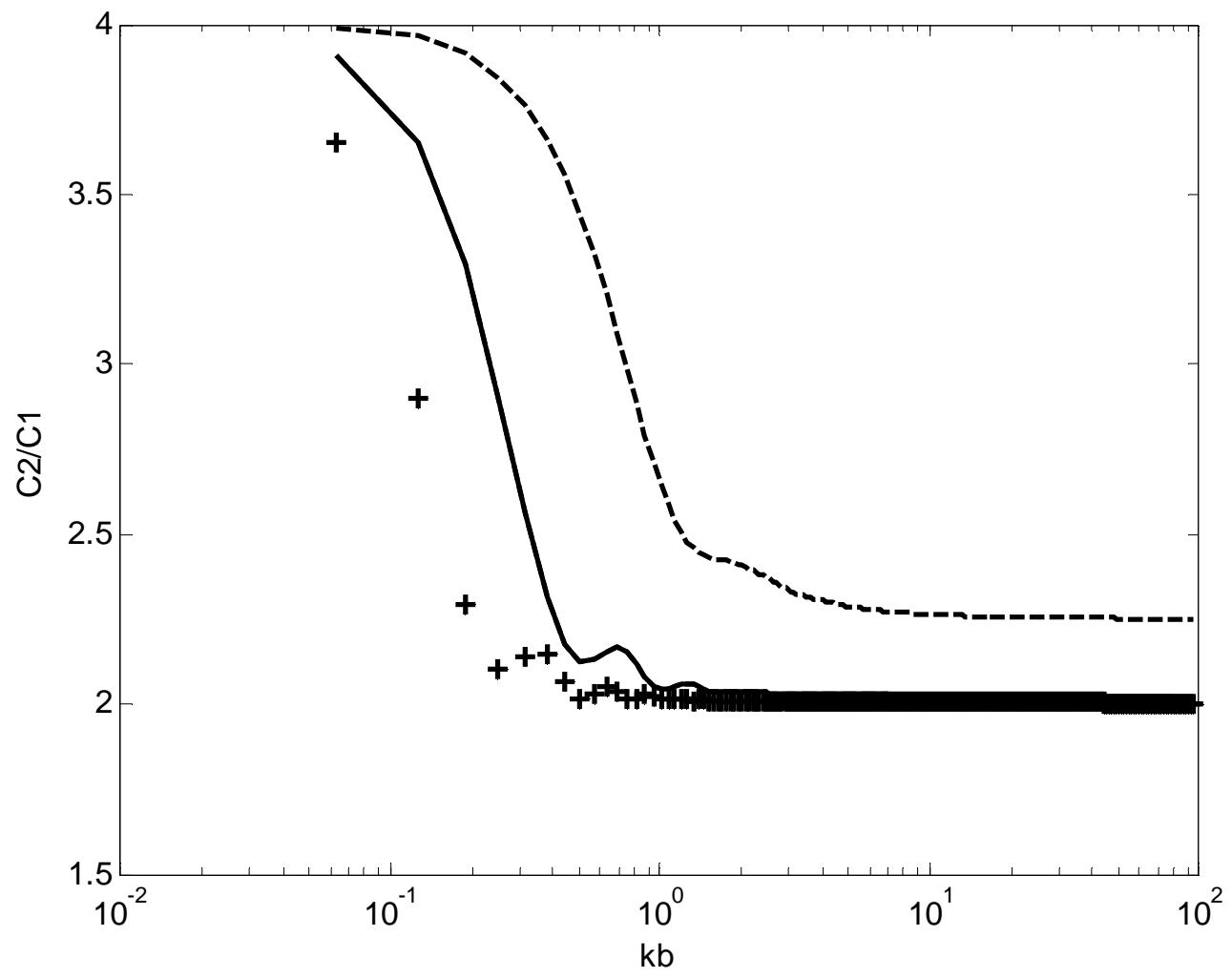

Fig. 4 


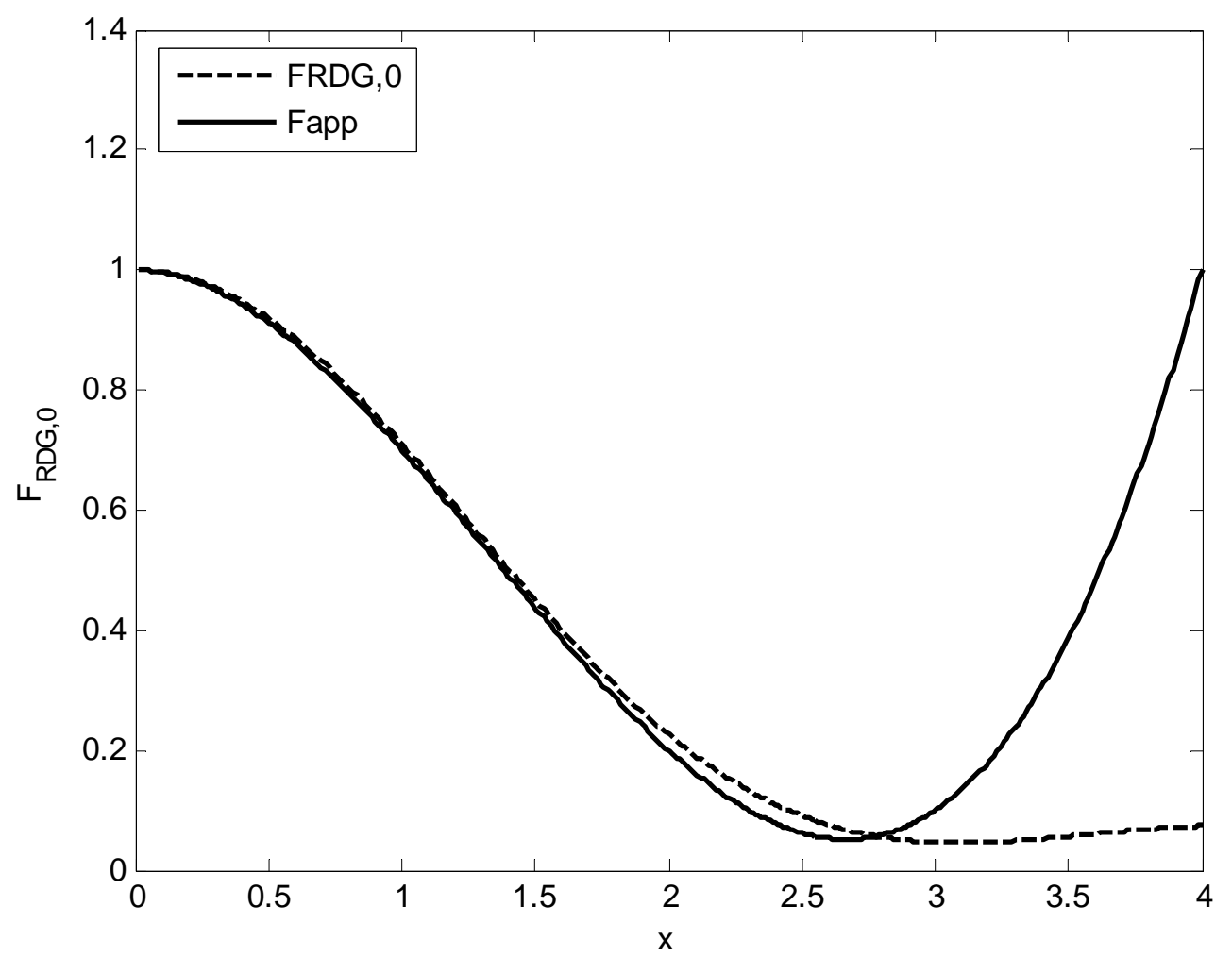

Fig.5 


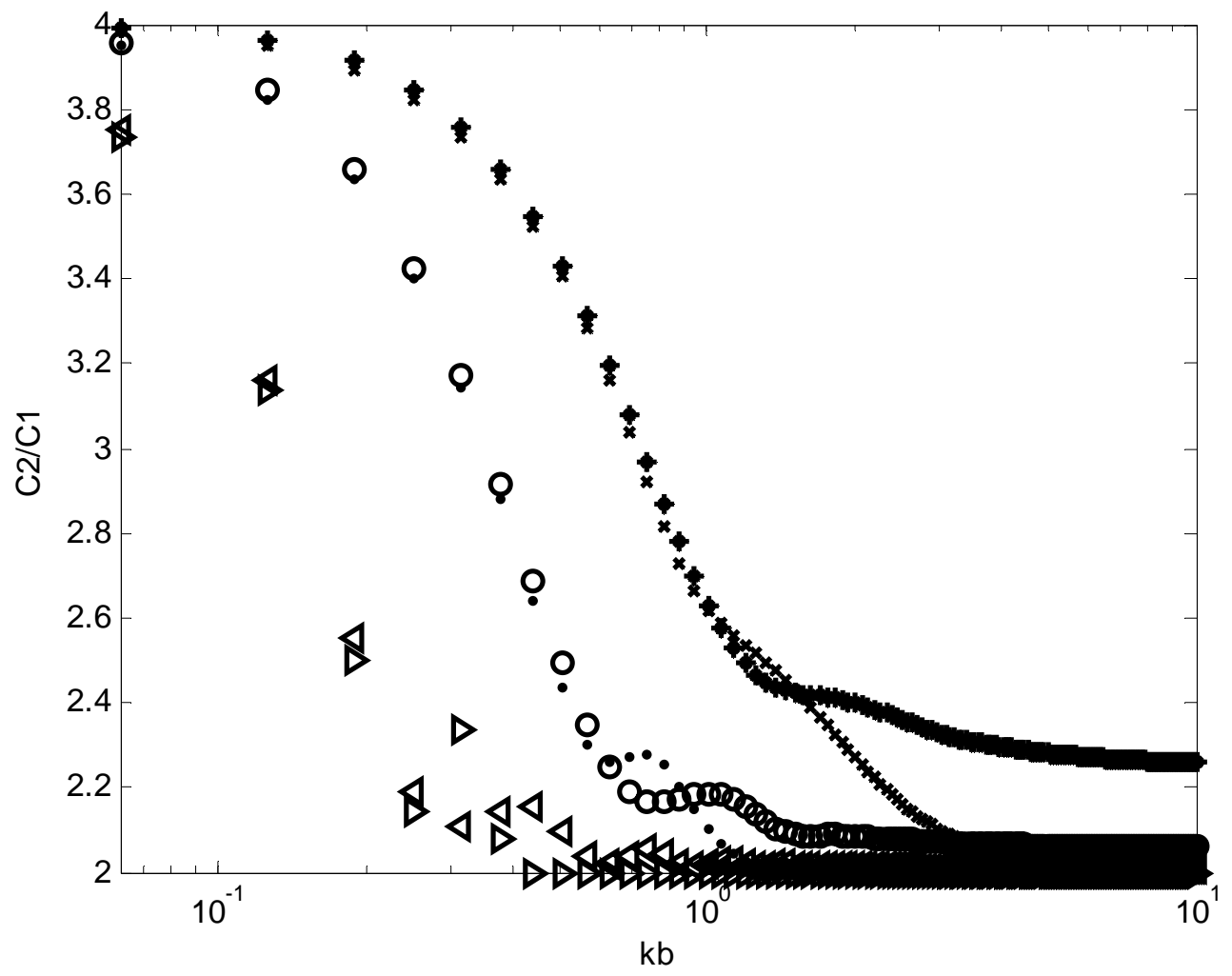

Fig. 6 


\begin{tabular}{|l|l|l|l|l|l|l|}
\hline $\mathrm{j}, \mathrm{m}$ & 0 & 1 & 2 & 3 & 4 & 5 \\
\hline 0 & 0 & 1 & & & & \\
\hline 1 & 0.4 & 0 & 1 & & & \\
\hline 2 & 0 & 1.2 & 0 & 1 & & \\
\hline 3 & 0.411 & 0 & 2.4 & 0 & 1 & \\
\hline 4 & 0 & 2.057 & 0 & 4 & 0 & 1 \\
\hline
\end{tabular}

Table 1: values of the coefficients $B_{m, j}$ for $\mathrm{j}<5$ 